$$
\text { "peschekschneider" — 2005/7/1 — 13:38 — page } 67 \text { — \#1 }
$$

\title{
Mathematische Bildung im Klagenfurter Doktorand(inn)enkolleg
}

\author{
Werner PescheK Und Edith Schneider
}

Abstract. In 2003 we set up a programme for PhD-studies („Doktorand(inn)enkolleg”) at the University of Klagenfurt which should promote and support PhD-studies in the field of mathematics education. Within this programme it is worked on the topic "general mathematics education" from different perspectives.

In the first part of this paper intentions, the fields of work and the form of organisation are briefly demonstrated. The second and main part considers in detail the work in one of the four fields of work, and finally, the third and last part presents some experiences with regard to the contents as well as general ones.

Key words and phrases: PhD-pogramme, general mathematics education, mathematics education, communication with experts, reflection, basic knowledge, information technology.

ZDM Subject Classification: A40, B50, C50, D30, U70.

\section{Das Klagenfurter Doktorand(inn)enkolleg}

Nach mehrjährigen Vorbereitungen auf institutioneller und konzeptioneller Ebene wurde an der Universität Klagenfurt (Österreich) ein viersemestriges Doktorand(inn)enkolleg (Promotionskolleg) eingerichtet, das sich vorrangig an im Beruf stehende Mathematiklehrer(innen) der Sekundarstufen I und II richtet und Doktoratsstudien im Fach Didaktik der Mathematik begleitend unterstützen soll. Das Kolleg wird von den Mitarbeiter(inne)n der Abteilung für Didaktik der Mathematik (ADM) durchgeführt.

Copyright (C) 2005 by University of Debrecen 


$$
\text { "peschekschneider" — 2005/7/1 — 13:38 — page } 68 \text { - \#2 }
$$

Mit der Einrichtung bzw. Durchführung dieses Doktorand(inn)enkollegs verfolgt die ADM insbesondere folgende Ziele (vgl. [4], [6]):

- Förderung des wissenschaftlichen Nachwuchses im Fach Didaktik der Mathematik

- Beiträge zur Professionalisierung von Lehrer(inne)n durch wissenschaftliche Denk-, Arbeits- und Reflexionsweisen sowie Kommunikationsformen

- engere inhaltliche und personelle Vernetzung zwischen universitärer Fachdidaktik und unterrichtlicher Praxis

- Erprobung eines Kooperations- und Interventionsmodells zwischen Fachdidaktiker-(inne)n an Universitäten und Lehrer(inne)n an Schulen

- Intensivierung empirischer Unterrichtsforschung

- Intensivierung der inhaltlichen Kooperationen innerhalb der ADM

Der inhaltliche Rahmen, in dem sich die Arbeiten im Kolleg bewegen, wird durch das Rahmenthema des Kollegs „Mathematische Bildung im informationstechnologischen Zeitalter" umrissen. Diese thematische Ausrichtung ergab sich als gemeinsamer Fokus der individuell verschiedenen Forschungsinteressen der Mitarbeiter(innen) der ADM und umfasst inhaltliche Aspekte mathematischer Bildung ebenso wie die kritische Reflexion formaler Methoden, Fragen nach gesellschaftlich erforderlichen mathematischen Kompetenzen in unserem informationstechnologischen Zeitalter ebenso wie epistemologische Grundfragen des Lernens von Mathematik und Fragen der Bedeutung informationstechnologischer Medien für das lernende Individuum. Konkret werden im Rahmen des Kollegs Dissertationen in folgenden Arbeitsgebieten betreut:

W. Dörfler: Entwicklung von mathematischem Wissen aus Handlungssystemen (epistemologische und semiotische Probleme des Mathematikunterrichts).

In diesem Arbeitsgebiet geht es insbesondere um die Entwicklung mathematischen Wissens aus Handlungssystemen anhand von so genannten „Lerntrajektorien”, um das Verhältnis von Begriffen und Symbolen bzw. von Verständnis und Rechnen beim Lernen von Mathematik, um die Qualität mathematischer Objekte und ihre kognitive, diskursive und soziale Entwicklung.

R. Fischer: Mathematik als Materialisierung des Abstrakten (sozialphilosophische Probleme des Mathematikunterrichts). 


$$
\text { "peschekschneider" — 2005/7/1 — 13:38 — page } 69 \text { — \#3 }
$$

Es geht in diesem Arbeitsgebiet vor allem um Untersuchungen zur Rolle der Mathematik in unserer von Informationstechnologien geprägten Gesellschaft, um eine Reflexion der gesellschaftlichen Bedeutung kollektiven mathematischen Wissens und formaler mathematischer Methoden (allgemeine Charakteristika der Mathematik, ihre Möglichkeiten und Grenzen) und um die Entwicklung entsprechender Reflexionskompetenzen im Mathematikunterricht.

G. Kadunz: Geometrie - Computer - Visualisierung.

Das Hauptaugenmerk ist hier auf die Verwendung von Darstellungen beim Lernen von (elementarer) Geometrie gerichtet, insbesondere wird das Verhältnis von Bild und Sprache sowie die Bedeutung von Zeichen in der Geometrie untersucht. Dabei wird immer auch die Rolle des Computers (insbesondere Dynamische Geometrie Software) und seiner Darstellungsmöglichkeiten mit reflektiert.

W. Peschek / Zum Bildungsgehalt mathematischer Inhalte: KommunikatiE. Schneider: onsfähigkeit als Orientierungsprinzip für einen allgemeinbildenden Mathematikunterricht.

Das Forschungsinteresse liegt hier vor allem in der Konkretisierung, Operationalisierung und curricularen Umsetzung sowie Evaluation eines von R. Fischer entwickelten Allgemeinbildungskonzepts, das sich nicht zuletzt auch als sehr geeignete bildungstheoretische Basis für einen computerunterstützten Mathematikunterricht erwiesen hat (vgl. [5]). - Auf dieses Arbeitsgebiet wird im zweiten Teil dieses Beitrags noch näher eingegangen.

Eine Besonderheit des Kollegs besteht darin, dass die jeweilige Forschungsarbeit der Doktorand(inn)en und das Verfassen der Dissertation in ein Bündel von eigens dafür konzipierten Lehrveranstaltungen eingebettet sind. Diese Veranstaltungen sollen sowohl der Beratung der individuellen Arbeiten als auch dem Vertrautwerden der Doktorand(inn)en mit verschiedenen didaktischen Forschungsgebieten und -methoden dienen. Weiters sollen dadurch der inhaltliche Austausch und die Kooperation unter den Doktorand(inn)en in besonderer Weise gefördert werden. Bei diesen Lehrveranstaltungen handelt es sich konkret um 


$$
\text { "peschekschneider" — 2005/7/1 — 13:38 — page } 70-\# 4
$$

- ein oder mehrere ein- bis zweitägige Forschungsseminare („Privatissima”) pro Semester zur gemeinsamen Arbeit in kleinen Forschungsgruppen sowie individuelle Beratungsgespräche mit dem jeweiligen Betreuer bzw. der jeweiligen Betreuerin

- mindestens eine zweitägige Lehrveranstaltung pro Semester zu einem ausgewählten didaktischen Themengebiet (Auswahl aus 2-3 verschiedenen Angeboten)

- ein viertägiges Forschungsseminar pro Studienjahr mit Präsentation und Diskussion der individuellen Arbeiten der Teilnehmer(innen) in der Gesamtgruppe sowie Nachbereitungen in den kleinen Forschungsgruppen.

Mit Rücksicht auf die schulische Unterrichtstätigkeit der Lehrer(innen) werden alle Lehrveranstaltungen in den Schulferien bzw. an Wochenenden abgehalten.

Eine weitere Besonderheit dieses Kollegs ist, dass die einzelnen Forschungsarbeiten nicht nur in den kleinen Forschungsgruppen (entsprechend den o. a. Arbeitsgebieten) diskutiert und aufeinander abgestimmt werden, sondern alle Arbeiten vom gesamten Betreuungsteam wahrgenommen, diskutiert und beraten werden. Mit dieser kollegialen Beratung im Betreuungsteam soll neben der Aushandlung wissenschaftlicher Standards innerhalb der ADM und der Sicherung der Qualität der einzelnen Arbeiten insbesondere auch eine besondere Beratungs- und Betreuungsqualität erreicht werden.

\section{Durchführung}

Nach einer österreichweiten Bewerbung des Kollegs im Wintersemester 2002/03 bekundeten 85 Mathematiklehrer(innen) aus ganz Österreich ihr Interesse an einer Teilnahme an diesem Kolleg; in einem aufwändigen Auswahlverfahren wurden schließlich jene 28 Kolleg(inn)en ermittelt, die in das Kolleg aufgenommen wurden. Mit einer Startveranstaltung im März 2003 wurde die Arbeit im Kolleg begonnen.

Im Jänner 2005 wurde das Kolleg von 22 Doktorand(inn)en erfolgreich abgeschlossen; von der Mehrheit dieser Doktorand(inn)en wird erwartet, dass sie ihre Dissertation und ihr Doktoratsstudium im Verlauf der nächsten 2-3 Semester erfolgreich beenden werden.

Die ADM plant, im Sommersemester 2006 mit einem neuen Durchgang des Doktorand(inn)enkollegs zu beginnen. 
"peschekschneider" — 2005/7/1 — 13:38 — page 71 — \#5

Mathematische Bildung im Klagenfurter Doktorand(inn)enkolleg

2. Kommunikationsfähigkeit als Orientierungsprinzip für einen allgemeinbildenden Mathematikunterricht

Wie bereits zuvor angesprochen, hat es sich eine Doktorand(inn)engruppe zur Aufgabe gemacht, ein von R. Fischer entwickeltes Konzept mathematischer (Allgemein-)Bildung zu konkretisieren, curricular umzusetzen und unterrichtspraktisch zu evaluieren. Dieses Arbeitsgebiet soll im Folgenden näher ausgeführt werden.

\subsection{R. Fischers Konzept der Höheren Allgemeinbildung}

Der Mathematikunterricht an weiterführenden Schulen (etwa ab dem 8. Schuljahr) lässt sich durch Verweise auf eine unmittelbare (direkte) Lebensvorbereitung kaum begründen, er kann allenfalls mittelbar zur Lebensvorbereitung beitragen. Für eine solche „Lebensvorbereitung im weiteren Sinne” ([3], S. 60) gibt es verschiedene Konzepte wie etwa jene der „formalen Bildung”, jene der „Schlüsselqualifikationen" oder auch das Allgemeinbildungskonzept von H. W. Heymann.

Vielen dieser Konzepte ist gemeinsam, dass sie den (mathematischen) Inhalt aus den Augen verlieren, dass fachliche Inhalte nur als Mittel für einen übergeordneten Zweck gesehen werden und diese Inhalte (und mit ihnen ganze Fächer) damit Gefahr laufen, beliebig und austauschbar zu werden. Das ist für jemanden, der an einen eigenständigen Bildungsgehalt mathematischer Inhalte glaubt - und das tun wir nahezu uneingeschränkt - natürlich keine sehr einladende bildungstheoretische Positionierung.

Wir gehen in unserem Ansatz von der sozialphilosophischen These aus, dass das Funktionieren einer hochdifferenzierten, arbeitsteilig organisierten, demokratischen Gesellschaft (wie der unseren) ganz wesentlich auf einem verständigen und emanzipierten Umgang mit hochspezialisiertem Expert(inn)enwissen basiert. Das setzt eine entsprechend hochentwickelte Kommunikation zwischen Expert(inn)en und Lai(inn)en voraus.

Die Fähigkeit zur Kommunikation mit Expert(inn)en hat eine fachlich-inhaltliche wie auch eine sozial-kommunikative Dimension:

Für Lai(inn)en wird es vor allem darum gehen, die richtigen Fragen an die Expert(inn)en zu stellen, die Antworten der Expert(inn)en zu gewichten und daraus Schlüsse zu ziehen (vgl. [1], S. 2f). Dafür sind zum Teil andere inhaltliche Kompetenzen erforderlich (und im Fachunterricht zu entwickeln) als jene, die man braucht, um selbst Experte oder Expertin zu werden. 
Zum anderen geht es aber auch um die soziale Interaktion zwischen Lai(inn)en und Expert(inn)en - die Kommunikation zwischen Lehrer(inne)n und Schüler(inne)n kann zum prägenden Modell für den gesellschaftlich erwünschten Umgang zwischen Expert(inn)en und Lai(inn)en werden (vgl. [3], S. 114).

Während H. W. Heymann ([3], S. 113f) stärker auf sozial-kommunikative Aspekte fokussiert, geht es R. Fischer vor allem um die fachlich-inhaltliche Seite: Er schlägt vor, die Fähigkeit zur Kommunikation mit Expert(inn)en als Orientierungsprinzip für die Auswahl von Inhalten an weiterführenden Schulen zu nehmen ([1], S. 5).

In einem ersten Konkretisierungsschritt identifiziert R. Fischer für die in einem Fach zu erwerbenden Kompetenzen folgende drei Bereiche (vgl. [1], S. 5):

- Grundkenntnisse und -fertigkeiten

(Konzepte, Begriffe, Darstellungsformen)

- Operieren

(zur Problemlösung oder zur Generierung neuen Wissens)

- Reflexion

(Möglichkeiten, Grenzen und Bedeutung von Begriffen und Methoden)

Während Expert(inn)en in allen drei Bereichen kompetent sein müssen, sieht R. Fischer für allgemeingebildete Lai(inn)en die Kompetenzbereiche Grundwissen und Reflexion als besonders bedeutsam an (vgl. Abbildung 1); er begründet dies mit den Anforderungen an eine gedeihliche Kommunikation mit Expert(inn)en: Grundwissen „,ist Voraussetzung für die Verständigung mit Experten”, Reflexion „,ist notwendig für die Beurteilung der Expertisen” ([1], S. 5).

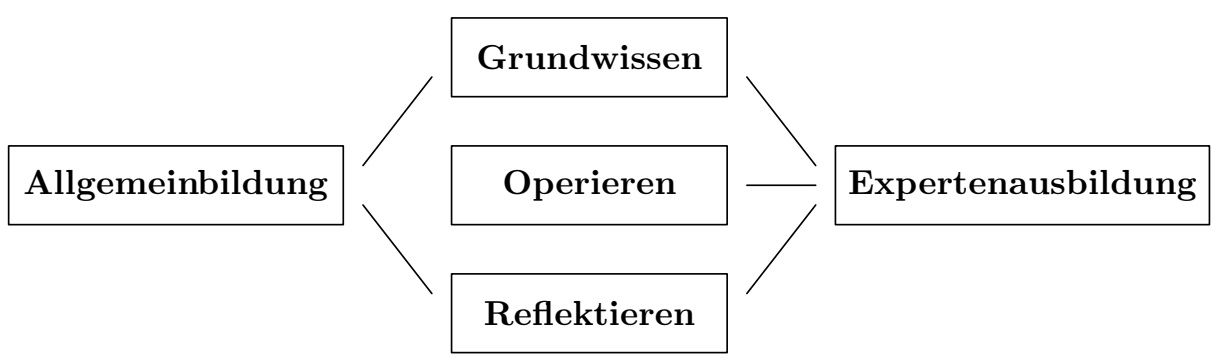

Abbildung 1. Kompetenzbereiche (nach [1], S. 5)

Diese für Expert(inn)en und Lai(inn)en unterschiedlichen Kompetenzprofile sollten dem Mathematikunterricht an weiterführenden Schulen eine Orientierung geben, die sich von der gegenwärtigen Realität nicht zuletzt durch eine „Reduktion 
"peschekschneider" — 2005/7/1 — 13:38 — page $73-\# 7$

der Ansprüche im Bezug auf Operieren und eine Erhöhung der Ansprüche im Bezug auf Reflexion" ([1], S. 6f) deutlich unterscheidet.

R. Fischers Orientierungsprinzip beschränkt sich aber nicht auf die Fähigkeit zur Kommunikation mit Expert(inn)en, sondern bezieht auch die Kommunikation mit der Allgemeinheit mit ein: Die durch den Besuch weiterführender Schulen „höher Gebildeten” (die „Intellektuellen”), „sollen in besonderer Weise befähigt sein, zwischen Experten und der, Allgemeinheit' zu vermitteln ... sie sollten das, was Experten meinen, in verständlicher Form erklären können, vor allem aber sollten sie in der Lage sein, Vorschläge für die Integration und Bewertung von Expertenmeinungen zu entwickeln" ([1], S. 3f). Eine derartige Kommunikationsfähigkeit scheint in zweifacher Hinsicht didaktisch von besonderem Interesse: Zum einen handelt es sich bei der Vermittlung zwischen Mensch und Wissen ja um ein originär didaktisches Anliegen, das hier fachspezifischer höherer Allgemeinbildung zugeordnet wird, zum anderen verweist die Forderung nach einer derartigen Kommunikationsfähigkeit auf unterrichtliche Intentionen und Interventionen, die bislang eher selten Gegenstand didaktischer Untersuchungen waren.

Die Forderung nach einer Reduktion des Operativen im Mathematikunterricht ist keineswegs neu. Soweit es die routinemäßigen operativen Tätigkeiten betrifft, ist diese didaktische Forderung so alt wie die (neuere) Mathematikdidaktik selbst. Durch die breite Verfügbarkeit von Computern und sehr leistungsfähiger mathematischer Software hat diese Forderung im letzten Jahrzehnt allerdings eine bemerkenswerte Renaissance erlebt - und nie zuvor hatte die Erfüllung dieser Forderung größere Realisierungschancen (vgl. [5], S. 29ff). Neu ist bei R. Fischer jedoch, dass er die Forderung nach Reduktion des Operativen in den Kontext eines universalen, sozialphilosophisch orientierten Allgemeinbildungskonzepts stellt, ja sie zum Kernstück seines Konzepts der Höheren Allgemeinbildung macht und damit begründet. Neu ist weiters auch der in diesem Allgemeinbildungskonzept verwendete Reflexionsbegriff, der vieles von dem zu erfassen scheint, was in der Mathematikdidaktik mit Analyse und Verständnis von Zusammenhängen, mit Bewertung, Metawissen u. Ä. bezeichnet wird, in mancher Hinsicht aber auch darüber hinaus zu gehen scheint. Neue, jedenfalls aber recht spezifische Aspekte scheinen schließlich auch die Begriffe des Grundwissens und der Grundkenntnisse zu beinhalten, die in R. Fischers Allgemeinbildungskonzept unter dem Blickwinkel einer sozialphilosophisch motivierten Kommunikationsfähigkeit gesehen werden (vgl. Abschnitt 2.4).
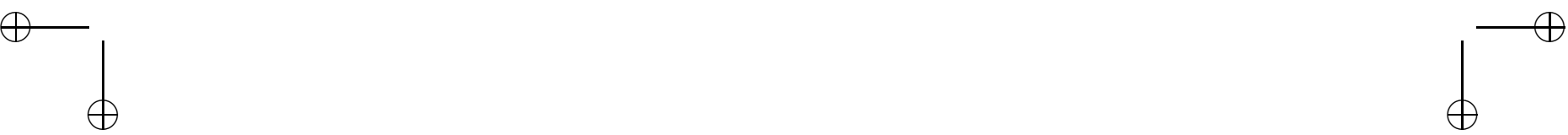


\subsection{Forschungsfragen und Entwicklungsaufgaben}

So einfach und überzeugend R. Fischers Konzept der Höheren Allgemeinbildung auf den ersten Blick erscheinen mag, es wirft eine Reihe von Fragen auf allen voran die Frage nach der curricularen Konkretisierung und der unterrichtlichen Umsetzung dieses Konzepts. Diese Frage bildet den Kern der Forschungsund Entwicklungsarbeiten in der von uns betreuten Doktorand(inn)engruppe; sie lässt sich in folgenden Forschungsfragen bzw. Entwicklungsaufgaben weiter konkretisieren:

(1) Was kann „Grundwissen” (also Grundkenntnisse und -fertigkeiten, die für eine gedeihliche Kommunikation mit Expert(inn)en und der Allgemeinheit unabdingbar sind) in einem schulmathematischen Themengebiet bedeuten?

(2) Was kann „Reflexion(swissen)” (wie es für die Bewertung und Integration von Expert(inn)enaussagen erforderlich ist) in einem schulmathematischen Themengebiet meinen?

(3) Wie lässt sich die Orientierung an der Kommunikationsfähigkeit mit Expert(inn)en und mit der Allgemeinheit curricular (in einem umfassenden Unterrichtskonzept) konkretisieren? Welche unterrichtlichen Sozialformen erscheinen angemessen? In welcher Weise können moderne Informationstechnologien die curricularen Intentionen unterstützen?

(4) In welcher Weise und in welchem Maße lassen sich die entwickelten curricularen Konzepte unterrichtspraktisch umsetzen? Welche Kompetenzen werden von den Schüler(inne)n in einem solchen Unterricht entwickelt?

(5) Welche Konsequenzen sind aufgrund der beobachteten Unterrichtsergebnisse für die curricularen Entwicklungen zu ziehen, welche Konsequenzen ergeben sich daraus für eine unterrichtspraktisch tragfähige und handhabbare Interpretation des Konzepts der Höheren Allgemeinbildung?

\subsection{Individuelle Arbeitsgebiete (Dissertationen)}

Die im vorangegangenen Abschnitt skizzierten Forschungsfragen und Entwicklungsaufgaben wurden bzw. werden von den sieben Doktorand(inn)en unserer Gruppe für folgende schulmathematische Themengebiete bearbeitet:

- Elementare Geometrie

- Funktionen

- Analytische Geometrie (und Lineare Algebra)

- Differenzialrechnung 
"peschekschneider" — 2005/7/1 — 13:38 — page 75 — \#9

- Systemdynamik

- Beschreibende Statistik

- Schließende Statistik

Durch jedes der genannten schulmathematischen Themengebiete ist ein individuelles Arbeitsgebiet (ein „Dissertationsthema”) eines Doktoranden bzw. einer Doktorandin gegeben. Innerhalb jedes individuellen Arbeitsgebietes wurden folgende Arbeitsschritte vereinbart und festgelegt:

\section{Analyse und Reflexion des gewählten Themengebiets}

Ausgangspunkt und Basis aller weiteren Arbeiten waren eine genaue Analyse und Reflexion des gewählten Arbeitsgebiets. Diese Analyse und Reflexion fokussierte auf den fachmathematischen Inhalt (Begriffe, Sätze, zentrale Ideen, Querverbindungen zu anderen mathematischen Inhalten, Arbeitsweisen), auf wichtige Anwendungsbereiche sowie auf vorliegende fachdidaktische Überlegungen dazu. Die Analysen und Reflexionen erfolgten insbesondere auch im Hinblick auf die Auslotung von Fähigkeiten, die zur Kommunikation innerhalb dieses Themengebiets und über dieses Themengebiet erforderlich erscheinen.

2. Identifikation von Grundwissen und Reflexion(swissen) innerhalb des gewählten Themengebiets

In einem zweiten Schritt wurde versucht, jenes Grundwissen herauszuarbeiten, zu begründen und in der Gruppe auszuhandeln, das für das gewählte Themengebiet besonders relevant erscheint sowie Reflexionsanlässe und Reflexionswissen zu identifizieren und in Aufgabenstellungen zu konkretisieren.

\section{Entwicklung eines Curriculums}

In weiterer Folge war ein Curriculum in Form einer umfassenden Unterrichtsplanung zu entwickeln. Dabei waren die im zweiten Schritt entwickelten Überlegungen zu Grundwissen und Reflexion(swissen) in geeigneter Weise in ein Unterrichtskonzept umzusetzen, das auf die Entwicklung einer entsprechenden Kommunikationsfähigkeit der Schüler(innen) fokussiert. Der sozialen Organisation des Unterrichts wurde dabei - neben den inhaltlichen Schwerpunktsetzungen besondere Bedeutung beigemessen, moderne Technologien sollten die Intentionen vor allem durch Materialisierungen mathematischen Wissens sowie als einfache Modelle „mathematischer Expert(inn)en” (vgl. [5], S. 36ff) unterstützen.

4. Unterrichtspraktische Erprobung und Evaluation des entwickelten Curriculums
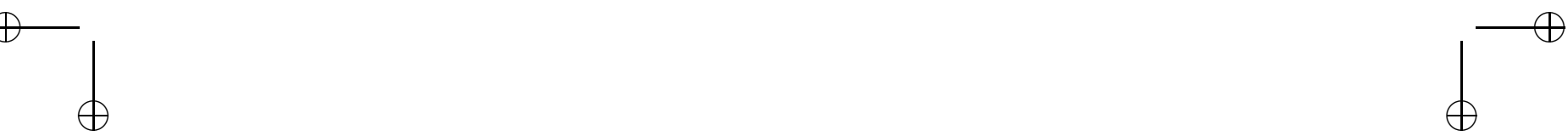


$$
\text { "peschekschneider" — 2005/7/1 — 13:38 — page } 76 \text { — \#10 }
$$

In einem vierten Schritt wird das entwickelte Curriculum unterrichtlich umgesetzt und einer Evaluierung unterzogen. Es geht dabei um die unterrichtspraktische Realisierbarkeit des entwickelten Curriculums, insbesondere aber um eine Analyse der Reflexionsprozesse sowie um eine Feststellung des von den Schüler(inne)n entwickelten Grund- und Reflexionswissens. (Die Mehrheit der Doktorand(inn)en ist derzeit, im Februar 2005, mit diesem Arbeitsschritt befasst.)

\section{Auswertung und Reflexion der Ergebnisse der unterrichtspraktischen Erpro- bung}

In einem letzten Arbeitsschritt schließlich werden die bei der unterrichtspraktischen Erprobung gesammelten Erfahrungen und die Ergebnisse der Evaluierung ausgewertet und im Hinblick auf das entwickelte Curriculum reflektiert; es sollen Vorschläge zur Revision des Curriculums dargelegt, allenfalls auch in einer entsprechenden Überarbeitung des Curriculums umgesetzt werden.

\subsection{Identifikation von Grundwissen und Reflexion(swissen)}

Grundwissen und Reflexion bilden die zentralen Kompetenzbereiche in R. Fischers Konzept der Höheren Allgemeinbildung. Entsprechend liegt ein zentrales Forschungsinteresse der Dissertationen bei der Identifikation von Grund- und Reflexionswissen bzw. bei der Auslotung von Reflexionsanlässen und Möglichkeiten innerhalb des bearbeiteten schulmathematischen Themengebiets. Dabei ist zunächst zu klären, wie Grundwissen und Reflexion(swissen) im Sinne R. Fischers Allgemeinbildungskonzepts verstanden werden können. Erst auf dieser Basis kann eine Identifikation von Grund- und Reflexionswissen und insbesondere dessen Aushandlung mit sich selbst und mit anderen erfolgen.

\subsubsection{Zur Interpretation von Grundwissen und Reflexion(swissen)}

Grundwissen umfasst nach R. Fischer ([2]) die aktive und passive Verfügbarkeit all jener Begriffe, Konzepte und Darstellungsformen, die für eine gedeihliche Kommunikation mit Expert(inn)en erforderlich sind; es umfasst somit also

- Wissen, das notwendig ist, um sich Expert(inn)en gegenüber verständlich machen zu können, verständliche Fragen an Expert(inn)en richten zu können, sowie

- Wissen, das notwendig ist, um Expert(inn)enantworten verständig erfassen und aufnehmen zu können. 
Eine Analyse so verstandenen Grundwissens (wie auch weitere Ausführungen dazu in [2]) macht sehr schnell deutlich, dass es sich bei diesem Grundwissen nicht nur um ein Grundvokabular zentraler mathematischer Begriffe und Konzepte sowie häufig verwendeter (z. B. symbolischer oder grafischer) Darstellungsformen handeln kann, sondern dass dieses Wissen immer auch verständige Grundvorstellungen über diese Begriffe, Konzepte und Darstellungsformen sowie deren charakteristische Anwendungsgebiete und -formen mit einschließt.

Weit komplexer und schwieriger ist die Frage, was Kommunikation mit Expert(inn)en denn überhaupt meint, wer dabei als Expertin oder Experte anzusehen ist und wie man in diesem Konzept überhaupt zu inhaltlichen Reduktionen kommen kann. Denn zweifellos ist die Kommunikation mit einem Experten oder einer Expertin in der Regel dann am ein-fachsten, wenn man selbst Expertin oder Experte ist und über entsprechendes Wissen verfügt.

Wir verstehen unter Kommunikation mit Expert(inn)en nicht nur das persönliche Gespräch, sondern ebenso die Auseinandersetzung mit materialisierten (z. B. in Literatur verschriftlichten oder in elektronischer Form repräsentierten) Aussagen und Argumentationen von Expert(inn)en. Weiters denken wir bei mathematischen Expert(inn)en nicht nur an Mathematiker(innen), sondern an all jene, die sich in ihren Darlegungen und Argumentationen mathematischer Begriffe und Methoden bedienen. Mit dieser Auffassung ist allerdings das Problem verbunden, dass sich daraus keine Anhaltspunkte für inhaltliche Abgrenzungen ergeben: Der Banachsche Fixpunktsatz kann unter Verweis auf die Kommunikation mit Expert(inn)en ebenso als Inhalt mathematischer Allgemeinbildung reklamiert werden wie das Kommutativgesetz der Addition natürlicher Zahlen. Tatsächlich sieht R. Fischer in der Kommunikationsfähigkeit zwar ein „Orientierungsprinzip für die Auswahl von Inhalten", aber kein eindeutiges, objektives Kriterium. Vielmehr sind entsprechende gesellschaftliche Aushandlungsprozesse und normative Entscheidungen erforderlich:

„Was man wissen und verstehen muß, um mit Experten verschiedenster Fachrichtungen kommunizieren zu können, ist nicht eindeutig und nicht objektiv ableitbar. Es ist von der Gesellschaft normativ festzulegen und damit eine Entscheidung. Anders ausgedrückt, es ist Ergebnis eines Prozesses der Aushandlung ..." ([1], S. 9)

Solche Aushandlungs- und Entscheidungsprozesse finden auf verschiedenen Ebenen der Gesellschaft und des Bildungswesens statt - in den (medialen) Diskussionen rund um die PISA-Ergebnisse, in Lehrplankommissionen, bei der Entwicklung, Approbation und Auswahl von Schulbüchern wie auch im konkreten 


$$
\text { "peschekschneider" — 2005/7/1 — 13:38 — page } 78 \text { — \#12 }
$$

Klassenzimmer (im ungünstigsten Fall durch Leistungsverweigerung). Kommunikationsfähigkeit mit Expert(inn)en und mit der Allgemeinheit kann ein Orientierungsprinzip für derartige Aushandlungs- und Entscheidungsprozesse (auf allen genannten Ebenen) sein, das geeignet erscheint, die Qualität derartiger Prozesse zu verbessern.

Unter Reflexion versteht R. Fischer ([1], [2]) das Nachdenken über Zusammenhänge, die objektsprachlich nicht im Vordergrund stehen. Er weist damit Reflexion als eine Denktätigkeit aus, die zunächst allerdings so allgemein ist, dass darunter fast jede Denktätigkeit jenseits erster intuitiver Wahrnehmung eines Gegenstandes subsummiert werden kann - vom Nachdenken darüber, ob 212 tatsächlich 24 ergibt, wie dies der Taschenrechner anzeigt, über Spekulationen, ob bei der nächsten Klassenarbeit auch das Lösen von Exponential-gleichungen abgeprüft werden wird bis hin zu Überlegungen, welche Bedeutung das Kulturgut Mathematik für uns als Individuum, für die Gesellschaft, in der wir leben und deren Bildung, oder für die Menschheit insgesamt haben könnte. Dieser letztgenannte Aspekt, der von R. Fischer besonders betont wird, fokussiert auf Fragen der Bewertung - und dabei im weitesten Sinne auf eine Bewertung des Verhältnisses zwischen Menschen, individuell oder kollektiv, und deren mathematischem Tun: Zum einen schreibt R. Fischer schon in seiner Begründung der besonderen Bedeutung des Kompetenzbereichs „Reflexion” für die Kommunikationsfähigkeit, dass Reflexion für die Beurteilung von Expert(inn)enaussagen notwendig sei, wobei er weniger die fachliche Richtigkeit der Expertise im Auge hat, als vielmehr deren Relevanz und Wichtigkeit im Hinblick auf Entscheidungen. Mit seinem Aushandlungskonzept geht R. Fischer aber noch einen Schritt weiter: Im Unterricht selbst und mit den Schülerinnen und Schülern, erst recht aber unter den Lehrenden (und letztlich noch allgemeiner in einem gesamt gesellschaftlichen Aushandlungsprozess), sollten explizite Auseinandersetzungen über Sinn und Bedeutung mathematischer Inhalte stattfinden und Unterschiede in der Bewertung von vorgeschlagenen Inhalten herausgearbeitet werden. Ablehnende Positionen müssen dabei zugelassen sein. „Gerade im Prozeß der Auseinandersetzung mit dem Vorgeschlagenen” meint Fischer ([1], S. 11), ,im Stellen der Frage: ,Was bedeuten die Inhalte für mich, was bedeuten sie für die Gesellschaft, was bedeuten sie für uns als Lerngemeinschaft (als Zwischenglied)?', vollzieht sich Bildung". Noch etwas pointierter formuliert R. Fischer an anderer Stelle:

„Die Zumutung des/r Lehrer/in als ExpertIn ist aber: Du mußt Mathematik lernen! Der Ansatz, Höhere Allgemeinbildung' beinhaltet, gerade darüber kritisch zu reflektieren." ([2], S. 31) 
"peschekschneider" — 2005/7/1 — 13:38 — page 79 — \#13

Die für R. Fischers Allgemeinbildungskonzept zentrale Forderung nach einer „Reduktion der Ansprüche im Bezug auf Operieren und eine Erhöhung der Ansprüche im Bezug auf Reflexion" ([1], S. 6f) ist daher wohl so zu verstehen, dass derartige Reflexionen im Unterrichtsgeschehen verstärkt explizit gemacht und kommuniziert, ihnen entsprechend Raum und vor allem Bedeutung eingeräumt werden sollen.

Das pädagogische Anliegen dabei ist, neben kognitiven Fähigkeiten zur Reflexion mathematischer Sachverhalte auch entsprechende Einstellungen und Haltungen (etwas genauer wissen oder verstehen wollen, sich selbst ein Bild machen oder ein Urteil bilden wollen ... ) weiter zu entwickeln, denn:

„Es reicht nicht aus, über Denkfertigkeiten und damit verbundenes Wissen zu verfügen, sondern es muß auch die Bereitschaft entwickelt werden, davon Gebrauch zu machen." ([3], S. 97)

Reflexion (als Prozess) kann aber nicht Selbstzweck sein bzw. bleiben. Mit der Forderung nach mehr Reflexion im Mathematikunterricht ist immer auch die Hoffnung auf deutliche inhaltliche Veränderungen des Mathematikunterrichts verbunden: Es soll verstärkt um ein Wissen über die vielfältigen Bezüge, Vernetzungen, Einschätzungen und Bewertungen gehen, die objektsprachlich nicht im Vordergrund stehen - wir sprechen in diesem Fall von Reflexionswissen. Die Elaboriertheit solchen Reflexionswissens kann als ein Gradmesser für die Qualität mathematischen Wissens und mathematischer Bildung gelten.

Reflexionswissen bedarf nicht notwendigerweise eigenständiger Reflexion. Zwar ist kaum zu bestreiten, dass es einen Unterschied macht, ob Wissen eigenständig oder rezeptiv entwickelt wurde, es erscheint jedoch sinnvoll, auch den verständigen Nachvollzug von Reflexionen anderer und die Entwicklung entsprechenden Wissens zum Reflexionswissen zu zählen, da man andernfalls ja jeden Kommunikations- und Aushandlungsprozess, bei dem man von Reflexionen anderer etwas lernt, als nicht zur Reflexion gehörig ansehen müsste.

Ein anderes Problem stellt sich bei der Abgrenzung des Reflexionswissens vom Grundwissen. So etwa wird man das Wissen um die absolut konstanten Veränderungen der linearen Funktion wohl zum Grundwissen zählen, selbst wenn es von den Schüler(inne)n durch eigenständige Reflexion des Begriffs der linearen Funktion entwickelt wurde. Reflexionswissen ist in diesem Fall (und wohl auch in vielen anderen Fällen) also zugleich auch Grundwissen. Wenn hier die Grenzen auch fließend und von den jeweiligen Vorkenntnissen abhängig sind, wie auch R. Fischer ([1], S. 5) vermerkt, kann es doch hilfreich sein, zu differenzieren: Wir sehen Reflexionswissen über Voraussetzungen, Möglichkeiten und Grenzen
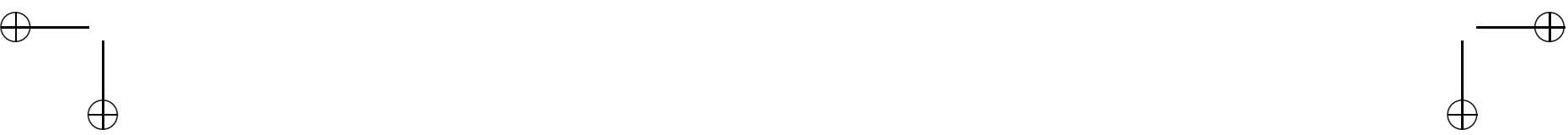


$$
\text { "peschekschneider" — 2005/7/1 — 13:38 — page } 80 \text { — \#14 }
$$

behandelter mathematischer Begriffe, Verfahren und Darstellungen ebenso wie entsprechendes Reflexionswissen über die Einordnung einzelner Wissensteile in ein zusammenhängendes Ganzes und dessen Bezüge zur Lebenswelt (Anwendungen) als unverzichtbaren Bestandteil qualitätsvollen, elaborierten Grundwissens. Ein derart reflektiertes Grundwissen sehen wir als Voraussetzung und Grundlage für bildungswirksame Aushandlungsprozesse über Sinn und Bedeutung dieses mathematischen Grundwissens und für eine emanzipierte (Selbst-)Reflexion der Beziehungen zwischen selbst erlebter Mathematik und dem eigenen Wertesystem.

2.4.2. Aushandlung mit sich selbst und mit anderen im Rahmen des Doktorand(inn)enkollegs

Den Ausgangspunkt der Arbeiten in unserer Doktorand(inn)engruppe bildete eine gründliche fachmathematische Analyse und Reflexion des jeweils gewählten schulmathematischen Themengebiets hinsichtlich zentraler Begriffe, Sätze, Verfahren und Zusammenhänge aus innermathematischer Sicht. Die Ergebnisse dieser Untersuchungen wurden verschriftlicht und innerhalb der Gruppe, teilweise auch mit fachmathematischen Expert(inn)en, diskutiert (woraus sich auch erste Hinweise darauf ergaben, welche Aspekte für eine Kommunikationsfähigkeit notwendig bzw. förderlich sein können).

In einem zweiten Schritt wurde versucht, typische inner- und außermathematische Anwendungen zu identifizieren und zu untersuchen, welches mathematische Wissen und Können erforderlich ist, um sich in diesen mathematischen Anwendungsbereichen kompetent bewegen und mit Hilfe der Mathematik kommunizieren zu können.

Basierend auf der fachmathematischen Analyse und der Analyse von Anwendungen wurde in einem dritten Schritt (auch unter Heranziehung fachdidaktischer Literatur) versucht, globale Ideen für dieses Themengebiet festzulegen. Unter ,globalen Ideen" werden dabei Metakonzepte (Bündel von Strategien, Handlungen, Verfahren, Techniken, Fragestellungen, Denk- und Arbeitsweisen) verstanden,

- die für ein größeres zusammenhängendes mathematisches Gebiet (mehrere Teilgebiete), deren Arbeitsweisen und Erscheinungsformen charakteristisch sind

- die die Entwicklung dieses mathematischen Gebiets nachhaltig beeinflusst haben

- die in inner- und außermathematischen Anwendungen dieses mathematischen Gebiets bedeutsam (leitend, sinnstiftend) sind 
- für die Entsprechungen im Alltagsdenken („Archetypen menschlichen Denkens") vorfindbar sind

- die geeignet erscheinen, curriculare Konstruktionen zu leiten, plausibel zu begründen und sinnstiftende Zusammenhänge zwischen lokalen Konzepten dieses mathematischen Gebiets herzustellen.

Globale Ideen charakterisieren eher allgemein und umfassend zentrale Anliegen eines Themengebiets, sie sagen sehr viel aus über typische Frage- und Problemstellungen dieses Gebiets, über inner- wie außermathematische Anwendungsmöglichkeiten, sie stellen einen sinnstiftenden Zusammenhang zwischen einzelnen Begriffen, Sätzen und Methoden des Themengebiets her und ermöglichen innerhalb dieses Rahmens eine Einordnung und Bewertung der lokalen Konzepte (Begriffe, Verfahren des jeweiligen Themengebiets). Reflexion in Bezug auf globale Ideen eines mathematischen Themengebiets bedeutet also immer auch, Beziehungen zwischen lokalen mathematischen Konzepten wie auch zu Anwendungen (Lebenssituationen) und zum Alltags denken herzustellen, lokale Konzepte in einem Gesamtzusammenhang zu positionieren und in diesem zu bewerten. In diesem Sinne kommt ihnen im Rahmen unserer Arbeit eine zentrale Bedeutung bei der Identifikation und Begründung von Grundwissen und Reflexion(swissen) zu.

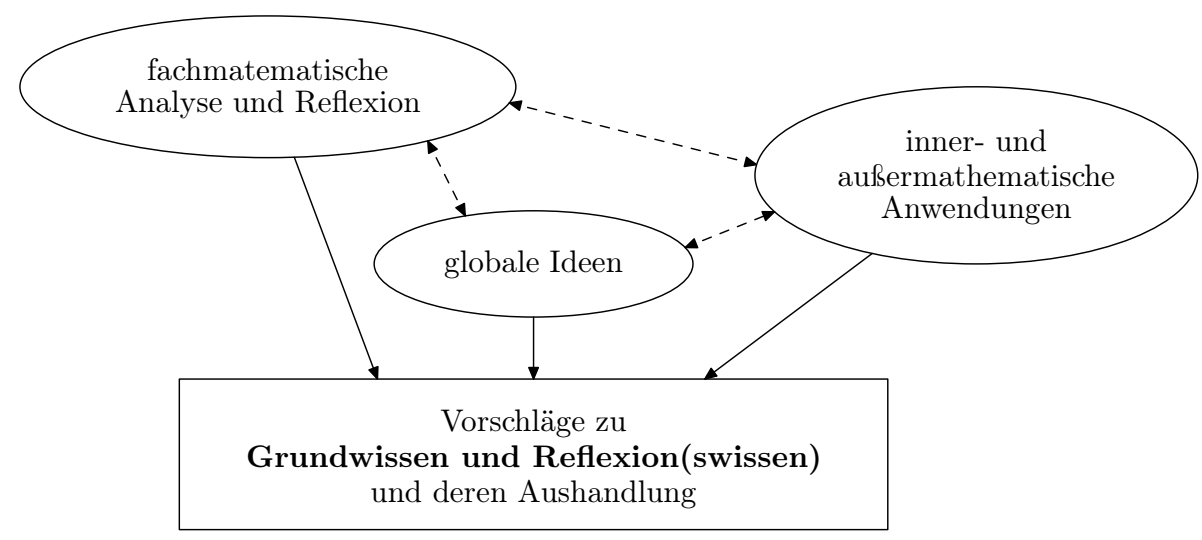

Abbildung 2. Idenifikation von Grundwissen und Reflexion(swissen)in einem mathematischen Themengebiet

Auf der Basis dieser Analysen wurde in der Folge versucht, Vorschläge für das zur Kommunikation mit Expert(inn)en und der Allgemeinheit erforderliche Grundwissen und zur Reflexion zu formulieren; die Vorschläge wurden innerhalb unserer Doktorand(inn)engruppe, zum Teil auch im Plenum des Kollegs diskutiert 


$$
\text { "peschekschneider" — 2005/7/1 — 13:38 — page } 82 \text { — \#16 }
$$

und ausgehandelt. Diese Aushandlungsprozesse verfolgten nicht nur das Ziel, sich als Lehrende(r) die für eine Curriculumkonstruktion erforderliche Klarheit über das angestrebte Grund- und Reflexionswissen zu verschaffen, es war damit vielmehr auch die Hoffnung verbunden, dass die Analysen und Reflexionen, die zu den Vorschlägen bezüglich Grund- und Reflexionswissen geführt hatten, wie auch die in den Aushandlungsprozessen angeführten Argumente und Gegenargumente und die Kommunikationsformen zumindest teilweise auch in die Curricula und den jeweiligen Unterricht Eingang finden würden.

\section{Einige Beobachtungen und Erfahrungen}

Ein Doktoratsstudium eines österreichischen Lehrers bzw. einer Lehrerin wird - im Gegensatz zu anderen Formen der Lehrer(innen)weiterbildung - weder zeitlich (durch Freistellungen für Lehrveranstaltungen, Reduktion der Lehrverpflichtung o. Ä.) noch finanziell (Ersatz von Fahrtoder Nächtigungskosten bzw. von Studiengebühren) unterstützt; ein erfolgreicher Studienabschluss wird innerhalb des Schulsystems weder finanziell noch in anderer Weise honoriert und führt auch kaum zu Verbesserungen der (geringen) Karrieremöglichkeiten von Lehrer(inne)n. Umso bemerkenswerter erscheint es uns, dass so viele Lehrer(innen) ernsthaftes Interesse an unserem Doktorand(inn)enkolleg gezeigt haben und das Kolleg von der überwiegenden Mehrheit der aufgenommenen Doktorand(inn)en trotz der hohen zeitlichen Belastung (ca. 10-15 Arbeitsstunden wöchentlich) erfolgreich absolviert werden konnte. Es ist damit zu rechnen, dass die Mehrheit dieser Absolvent(inn)en in den nächsten 2-3 Semestern ihr Doktoratsstudium erfolgreich abschließen wird.

Die fachdidaktische Ausbildung im Rahmen eines universitären Lehramtsstudiums ist in Österreich eher gering (Pflichtlehrveranstaltungen im Ausmaß von ca. 10-20 Semester-wochenstunden) und fokussiert in der Regel auf eine schulpraktische Ausbildung. Mangelndes fachdidaktisches Vorwissen und eine nur geringe wissenschaftspropädeutische Vorbildung und Vorerfahrung erschweren natürlich den Zugang zu wissenschaftlichen Arbeiten in diesem Fach. Insbesondere bei der Verschriftlichung wissenschaftlicher Argumentationen und beim Verfassen wissenschaftlicher Texte zeigten sich im Kolleg anfangs Schwierigkeiten.

Bei der Mehrheit unserer Doktorand(inn)en handelt es sich um sehr erfahrene Lehrer(innen). Das bringt neben vielen Vorteilen gelegentlich auch den Nachteil mit sich, dass es den Lehrer(inne)n manchmal schwer fällt, sich von vertrauten inhaltlichen Schwerpunktsetzungen oder Methoden zu lösen, sich auf neue und 
ungewohnte Wege einzulassen. Dies insbesondere dann, wenn - wie etwa in unserer Gruppe - davon ein größeres schulmathematisches Themengebiet curricular betroffen ist.

Österreichische Lehrer(innen) sind in ihren inhaltlichen Orientierungen und Schwerpunktsetzungen (innerhalb eines durch den Lehrplan vorgegebenen Rahmens) weitgehend autonom und kaum daran gewöhnt, ihre diesbezüglichen Bewertungen und Entscheidungen ernsthaft zur Diskussion zu stellen. R. Fischer sieht aber gerade in Aushandlungsprozessen über inhaltliche Schwerpunktsetzungen (unter Lehrer(inne)n, ganz besonders aber auch mit Schüler(inne)n!) einen zentralen und konstitutiven Bestandteil seines Allgemeinbildungskonzepts. Dies wurde und wird in der von uns betreuten Gruppe gelegentlich schon auch als besondere Belastung empfunden, sowohl emotional (bei der eigenen Arbeit) als auch im Hinblick darauf, dass immer auch alle Arbeiten der Kolleg(inn)en sehr gewissenhaft zur Kenntnis genommen und diskutiert werden müssen. Die Übertragung solcher Aushandlungsprozesse in den eigenen Unterricht erfolgt(e) aus unserer Sicht teilweise etwas zögerlich.

Die Arbeit in unserer Gruppe ist als gemeinsames Forschungsvorhaben konzipiert, an dem kooperativ gearbeitet wird. Die Vorteile einer derartigen Organisation liegen für die einzelne Dissertantin bzw. den einzelnen Dissertanten in der kollegialen Beratung und sozialen Aufgehobenheit in der Gruppe, in der Einbettung der eigenen Arbeit in einen größeren Forschungszusammenhang und ganz besonders natürlich in der Nutzung inhaltlicher Synergien bei der Bearbeitung der Themen wie auch bei der Nutzung der jeweiligen Ergebnisse. Als Nachteil mussten wir zur Kenntnis nehmen, dass eine solche Organisation wechselseitige Abhängigkeiten schafft und eine recht strenge Einhaltung gemeinsam festgelegter zeitlicher Arbeitspläne erfordert, was sich gelegentlich für die/den Einzelne(n) als nicht sinnvoll oder nicht möglich erweist. (In den anderen Gruppen des Kollegs sind die einzelnen Arbeiten weniger auf einander bezogen und daher die wechselseitigen Abhängigkeiten deutlich geringer.)

Als nicht zu unterschätzendes Problem hat sich schließlich auch die Doppelrolle unserer Dissertant(inn)en im konkreten Unterricht erwiesen: Die Rolle als Forscher(in) verlangt oftmals auch dann eine Beschränkung auf distanzierte Beobachtung, wenn vom Lehrer bzw. der Lehrerin Interventionen bzw. Hilfestellungen erwartet oder eingefordert werden. 


$$
\text { "peschekschneider" — 2005/7/1 — 13:38 — page } 84 \text { — \#18 }
$$

\section{Literatur}

[1] R. Fischer, Höhere Allgemeinbildung, Typoskript, Univ. Klagenfurt/IFF Wien, 15 S.

[2] R. Fischer, Höhere Allgemeinbildung II, Typoskript, Univ. Klagenfurt/IFF Wien, 46 $\mathrm{S}$.

[3] H. W. Heymann, Allgemeinbildung und Mathematik, Beltz, Weinheim und Basel, 1996.

[4] W. Peschek, Lehrer(innen) als Forscher(innen) - Das Klagenfurter Doktorand(inn)enkolleg, Zentralblatt für Didaktik der Mathematik 36 (1) (2004), 9-14.

[5] E. Schneider, Computeralgebrasysteme in einem allgemeinbildenden Mathematikunterricht, Didaktische Orientierungen - Praktische Erfahrungen, Profil, München und Wien, 2002.

[6] E. Schneider, Das Klagenfurter Doktorand(inn)enkolleg - ein Kooperationsmodell zwischen Fachdidaktiker(inne)n und Schulpraktiker(inne)n, in: Beiträge zum Mathematikunterricht, (H.-W. Henn, ed.), Franzbecker, Hildesheim und Berlin, 2003, S. 497-500.

WERNER PESCHEK UND EDITH SCHNEIDER

ABTEILUNG FÜR DIDAKTIK DER MATHEMATIK (ADM)

ALPEN-ADRIA-UNIVERSITÄT KLAGENFURT

UNIVERSITÄTSSTR. 65

A-9020 KLAGENFURT

AUSTRIA

E-mail: werner.peschek@uni-klu.ac.at

E-mail: edith.schneider@uni-klu.ac.at

(Received February, 2005) 